\title{
Effects of excitation light intensity on parathyroid autofluorescence with a novel near-infrared fluorescence imaging system: two surgical case reports
}

\author{
Mariko Aoyama ${ }^{1}$, Hiromitsu Takizawa ${ }^{1}$, Kiyoshige Yamamoto ${ }^{1}$, Tomohiro Inui ${ }^{1}$, Naoki Miyamoto ${ }^{1}$, \\ Shinichi Sakamoto ${ }^{1}$, Tomoko Kobayashi ${ }^{2}$, Hisanori Uehara ${ }^{2}$, Akira Tangoku ${ }^{1}$ \\ ${ }^{1}$ Department of Thoracic, Endocrine Surgery, and Oncology, Institute of Health Biosciences, The University of Tokushima, Kuramoto-cho, \\ Tokushima, Japan; ${ }^{2}$ Department of Pathology, Tokushima University Hospital, Kuramoto-cho, Tokushima, Japan \\ Correspondence to: Hiromitsu Takizawa. Department of Thoracic, Endocrine Surgery, and Oncology, Institute of Health Biosciences, The University \\ of Tokushima, 3-18-15, Kuramoto-cho, Tokushima, 770-8503, Japan. Email: takizawa@tokushima-u.ac.jp.
}

\begin{abstract}
The intraoperative identification and preservation of the parathyroid glands are vital techniques, which are largely dependent on a surgeon's experience. Therefore, a simple and reproducible technique to identify the parathyroid glands during surgery is needed. Parathyroid tissue shows near-infrared (NIR) autofluorescence, which enables the intraoperative identification of the parathyroid gland. We herein present two cases that underwent surgery on the parathyroid glands, which were observed using the NIR fluorescence imaging system LIGHTVISION ${ }^{\circledR}$ (Shimazu, Kyoto, Japan). In a case of papillary thyroid carcinoma, the system was adopted to preserve normal parathyroid glands during left hemithyroidectomy. The left lower parathyroid gland was identified using the imaging system under white light; however, its autofluorescence was visualized more clearly with the excitation light of NIR. In a case of primary hyperparathyroidism due to MEN1, the system was adopted to identify and remove all of the parathyroid glands during total parathyroidectomy. The autofluorescence of diseased glands was weaker than that of normal glands, even with the excitation light of NIR. When the parathyroid glands were irradiated with a red laser pointer, the intensity of autofluorescence significantly increased. However, the largest gland, which was pathologically proven to contain strongly proliferating chief cells, did not show autofluorescence. These results suggest that normal or less diseased parathyroid glands, which are generally small and difficult to identify during surgery, showed relatively strong autofluorescence. A stronger excitation light increases the autofluorescence of parathyroid glands, which enhances sensitivity for detecting parathyroid glands during surgery. In conclusion, LIGHTVISION ${ }^{\circledR}$ is a useful device to identify parathyroid glands and an additional excitation light of a red laser pointer increases the detection sensitivity.
\end{abstract}

Keywords: Autofluorescence; parathyroid gland; near-infrared; surgery

Submitted Mar 24, 2020. Accepted for publication Jul 19, 2020.

doi: $10.21037 /$ gs-20-386

View this article at: http://dx.doi.org/10.21037/gs-20-386

\section{Introduction}

The intraoperative identification and preservation of the parathyroid glands are vital in thyroid and parathyroid surgeries. During thyroidectomy, the careful preservation of the parathyroid glands is important due to temporary or permanent hypocalcemia caused by hypothyroidism.
Diseased parathyroid glands need to be accurately identified and removed during surgery because their incomplete excision results in recurrence. Due to their small size and various anatomical positions, difficulties are associated with distinguishing the parathyroid glands from surrounding tissues, such as the lymph nodes and adipose tissue (1).

Surgeons continue to rely on visual assessments during 
surgery in order to identify and preserve the parathyroid glands, and the success of this approach is largely dependent on a surgeon's experience (2). Therefore, a simple and reproducible technique to identify the parathyroid glands during surgery is needed.

In 2011, Paras et al. showed that the parathyroid glands possessed a fluorescent property in the near-infrared (NIR) region and emitted NIR light at a peak wavelength of $820 \mathrm{~nm}$ when illuminated with light at $785 \mathrm{~nm}$ (3). McWade et al. conducted imaging of parathyroid gland autofluorescence during human thyroidectomy for the first time in 2014 (4). Autofluorescence spectroscopy is an effective technique for intraoperatively visualizing the parathyroid glands $(5,6)$.

LIGHTVISION $^{\circledR}$ (Shimazu, Kyoto, Japan) is a single monitor that evaluates NIR as well as visible light images and three-dimensional images superimposed on them in real-time. The xenon light source of the system provides visible light at a wavelength of 780-800 $\mathrm{nm}$ and NIR excitation light at a wavelength of $800-850 \mathrm{~nm}$. Previous studies reported that it was possible to confirm indocyanine green (ICG)/NIR fluorescence without turning off the operating room light in evaluations of vascularized lymph node flaps (7) and lymphography for lymphaticovenular anastomosis (8).

We herein demonstrate the effectiveness of the excitation light to autofluorescence in the intraoperative identification of the parathyroid glands using the novel NIR fluorescence imaging system LIGHTVISION ${ }^{\circledR}$. We also investigated differences in autofluorescence by the histological background.

The following cases were presented in accordance with the CARE guidelines (9). Available at http://dx.doi. org/10.21037/gs-20-386.

\section{Case presentation}

\section{Case 1}

A 33 -year-old female with a mass of approximately $2.0 \times 1.0 \times 3.0 \mathrm{~cm}$ in the left thyroid lobe was diagnosed with papillary thyroid carcinoma. She had no symptoms and she had no previous or family history. An ultrasound examination showed a mass of approximately $2 \mathrm{~cm}$ in diameter in the left thyroid lobe and the absence of swollen parathyroid glands. Her preoperative serum calcium level was within the normal range. She underwent left hemithyroidectomy and cervical lymph node dissection. The left upper parathyroid gland was easily identified without observations of autofluorescence. After lateral mobilization of the left thyroid lobe and exposure of the recurrent laryngeal nerve, LIGHTVISION ${ }^{\circledR}$ was employed to locate the left lower parathyroid gland. Autofluorescence was observed in the parathyroid gland under white light, and became stronger with NIR excitation light. When adipose tissue was removed from the surface of the parathyroid glands, the intensify of autofluorescence increased. Furthermore, autofluorescence in the parathyroid glands was stronger ex vivo than in vivo (Figure 1). Resected tissue was pathologically diagnosed as normal parathyroid glands and implanted into the left sternocleidomastoid muscle. She had no tetany and her postoperative serum $\mathrm{Ca}^{2+}$ level was within the normal range.

\section{Case 2}

A 47-year-old male was referred to our hospital with a high serum $\mathrm{Ca}^{2+}$ level $(11.1 \mathrm{mg} / \mathrm{dL})$. He had no symptoms. His sister had been diagnosed with MEN1. Laboratory tests revealed an elevated intact parathyroid hormone $(\mathrm{PTH})$ level $(664 \mathrm{pg} / \mathrm{mL})$. Neck ultrasonography showed one enlarged parathyroid gland behind the upper pole of the left thyroid lobe, and 99m-Technetium Methoxy-IsobutylIsonitrile (MIBI) scintigraphy revealed uptake in both the upper and lower left thyroid lobes. He was diagnosed with primary hyperparathyroidism due to MEN1 and underwent total parathyroidectomy.

The parathyroid glands were removed during surgery. The left upper gland was the largest, weighing $8,720 \mathrm{mg}$. Although autofluorescence was weaker than that in Case 1, it was observed in 5 parathyroid glands, but was absent in the left upper gland. To compare the intensity of autofluorescence between the parathyroid glands and surrounding tissues, light was irradiated to the parathyroid glands, thyroid tissues, lymph nodes, adipose tissue, and thymus tissue. Differences in the intensity of autofluorescence were small. When the parathyroid glands were irradiated with a red laser pointer ex vivo, the intensity of autofluorescence significantly increased and the difference in fluorescence intensity from the surrounding tissues was more clearly confirmed (Figure 2A). However, autofluorescence was not observed in the largest gland, even with red laser pointer excitation (Figure $2 B$ ). In the postoperative examination, serum $\mathrm{Ca}^{2+}$ and intact $\mathrm{PTH}$ levels decreased (serum $\mathrm{Ca}^{2+}: 7.4 \mathrm{mg} / \mathrm{dL}$ and intact PTH: $9 \mathrm{pg} / \mathrm{mL}$ ). Calcium and vitamin $\mathrm{D}$ were replenished, but continued to be replenished one year after surgery. 

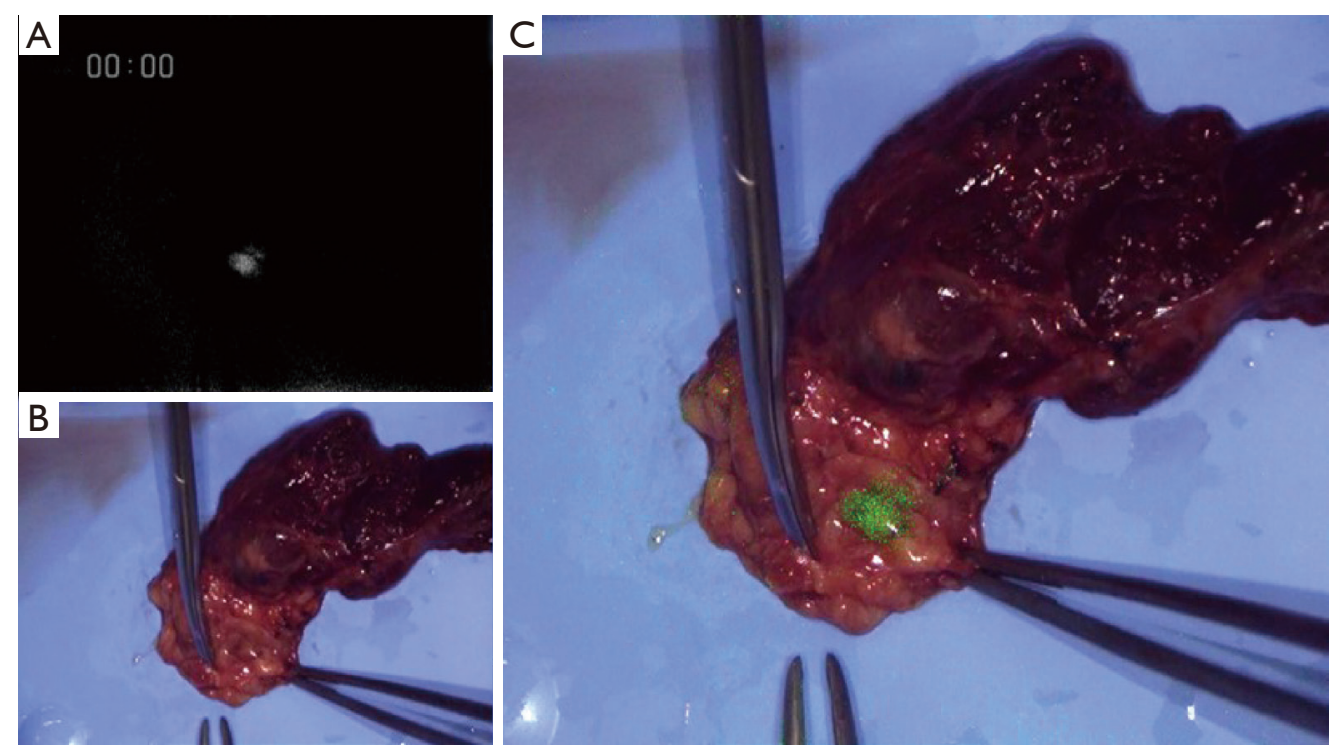

Figure 1 Autofluorescence in the normal left lower parathyroid gland. When adipose tissue was removed from the surface of the parathyroid glands, autofluorescence was more clearly observed. (A) NIR light image; (B) visible light image; (C) three-dimensional images superimposed on NIR and visible light images. NIR, near infrared.

A histological examination revealed the proliferation of chief cells and eosinophilic cells in all glands, diagnosed as hyperplasia (Figure 3). In parathyroid glands other than the upper parathyroid gland, adipose tissue was maintained, but at smaller amounts than the normal parathyroid glands (Figure $3 A$ ). The left upper gland contained less adipose tissue and more strongly proliferating chief cells (Figure $3 B$ ).

All procedures performed in studies involving human participants were in accordance with the ethical standards of the institutional and/or national research committee(s) and with the Helsinki Declaration (as revised in 2013). Written informed consent was obtained from the patient.

\section{Discussion}

In Case 1, autofluorescence was stronger with NIR excitation light. Autofluorescence was weak when adipose tissue was present on the surface of the parathyroid glands. Autofluorescence in the parathyroid glands was stronger ex vivo than in vivo. These results indicated that strong excitation light is needed to increase autofluorescence in the parathyroid glands. A technique that increases the intensity of excitation light to enhance the sensitivity of detecting autofluorescence in the parathyroid glands was considered to be necessary. In Case 2, a red laser pointer was employed to increase the energy of excitation light, and stronger autofluorescence was observed in the parathyroid glands. These results indicated that the light of a red laser pointer has the same wavelength as the excitation light of LIGHTVISION ${ }^{\circledR}$, and the addition of excitation light increases the visibility of autofluorescence in the parathyroid glands.

In order to increase intraoperative identification rate of the parathyroid glands by autofluorescence, stronger excitation light was considered to be necessary. A limitation of autofluorescence spectroscopy of the parathyroid glands is the difficulty associated with observing autofluorescence in deeply located parathyroid glands because the intensity of fluorescence decreases with increases in the distance from the parathyroid glands to the surface $(4,10)$. As a method to strengthen the excitation light, Ladurner et al. previously reported that a higher gain setting of the camera's blue channel during NIR autofluorescence imaging may effectively apply a stronger light source; however, this method may cause noise (11). We used a red laser pointer to enhance the excitation light. Our method is very simple, and images of autofluorescence in the parathyroid glands may be visualized without extra gain. However, further studies are needed to confirm the effectiveness of this method because this was a preliminary study.

Autofluorescence was observed in the majority of the parathyroid glands in Case 2, but was weaker than that 

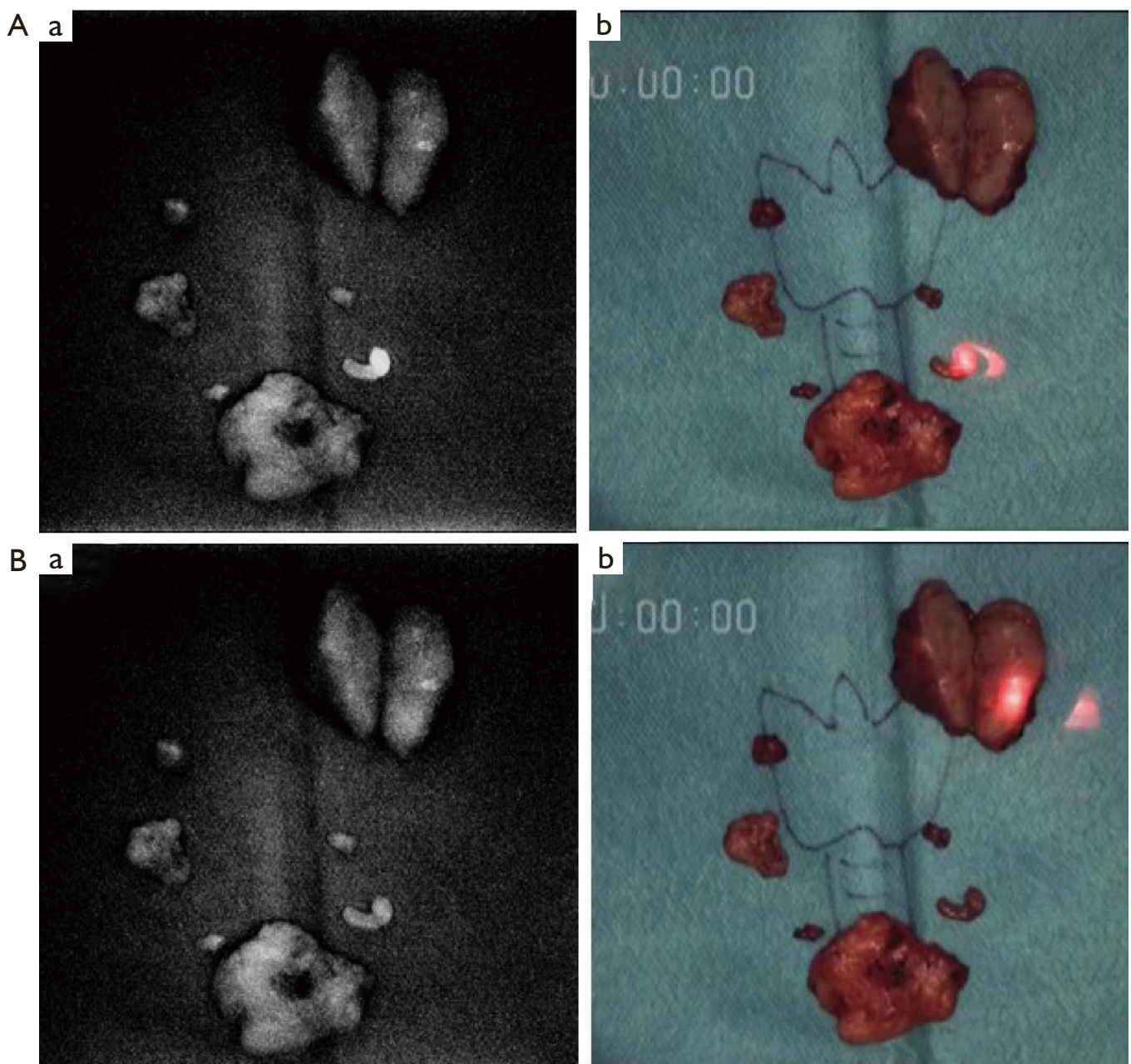

Figure 2 Autofluorescence in parathyroid glands of a MEN1 patient. The intensity of autofluorescence in the parathyroid glands increased with red laser pointer excitation ex vivo, whereas that in the largest gland did not. (A) The left lower gland within the cervical thymus; (B) the left upper gland: a, NIR light image; b, visible light image.

in Case 1. The largest parathyroid glands showed weak or no autofluorescence even with excitation by the red laser pointer. In the present cases, autofluorescence was weaker in diseased than in normal parathyroid glands. However, Falco et al. reported that parathyroid adenomas showed significantly stronger fluorescence than normal parathyroid glands (12). Furthermore, Ladurner et al. found no significant differences in autofluorescence between parathyroid adenomas, hyperplasia, and normal parathyroid glands (6). The mechanisms underlying autofluorescence in the parathyroid glands currently remain unclear. Furthermore, disease-dependent changes in autofluorescence in these glands have not yet been examined in detail. In the histological examination, the largest gland contained the most strongly proliferating chief cells, while other parathyroid glands showed mild proliferation. The proliferation of parathyroid gland cells may affect the intensity of autofluorescence observed. Therefore, the method described herein may be an effective tool for examining normal parathyroid glands or parathyroid glands with few pathological changes.

Previous studies reported that LIGHTVISION $^{\circledR}$ is an effective tool for evaluating vascularized lymph node flaps (7) and lymphography for lymphaticovenular anastomosis (8). This is the first study to investigate autofluorescence in the parathyroid glands using LIGHTVISION ${ }^{\circledR}$. One of the advantages of LIGHTVISION ${ }^{\circledR}$ is that autofluorescence in the parathyroid glands may be confirmed without 

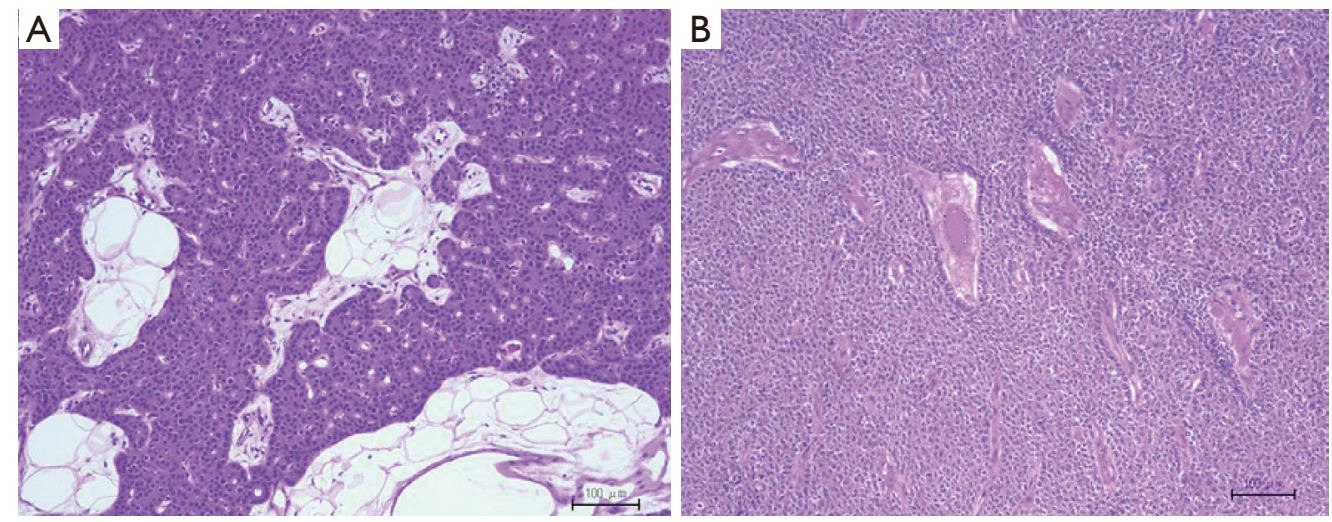

Figure 3 Histological findings. Adipose tissue was maintained in the left lower gland, while the left upper gland contained less adipose tissue and more strongly proliferating chief cells. (A) The left lower gland within the cervical thymus; (B) the left upper gland (HE stain, scale bar: $100 \mu \mathrm{m})$.

turning off the operation room light. In previous studies, the operation room light needed to be turned off in order to obtain NIR autofluorescence images $(4,10)$. In our method, autofluorescence in the parathyroid glands may be confirmed while the operation room light remains on, which may increase the accuracy of surgical procedures. As another advantage of LIGHTVISION ${ }^{\circledR}$, NIR and visible light images as well as three-dimensional images superimposed on them may be evaluated on a single monitor in real-time. Kim et al. introduced a technique to visualize the parathyroid glands and surrounding tissues in a single image using a 780-nm collimated light-emitting diode (Thorlabs, Newton, NJ, USA) with an excitation filter appropriate for parathyroid gland autofluorescence, an illuminator (INFRALUX-300, Daekyoung, Korea) for reflection of the entire surgical area, and a digital single-lens (Canon, EOSREBEL T3, Japan) with a camera lens (Canon EF $50 \mathrm{~mm} \mathrm{f} / 1.8 \mathrm{II}$, Japan) including an emission filter $(13,14)$. In our method, the same image may be more easily obtained by switching buttons. This novel imaging system may be an effective tool for the intraoperative identification of the parathyroid glands.

Our method may be useful for the intraoperative identification of normal parathyroid glands during thyroidectomy and slightly enlarged parathyroid glands that cannot be confirmed by ultrasonography or $99 \mathrm{~m}$-Technetium MIBI scintigraphy. A red laser pointer may also be an effective tool for easily enhancing excitation light and increasing the detection sensitivity of autofluorescence.

\section{Acknowledgments}

Funding: None.

\section{Footnote}

Reporting Checklist: The authors have completed the CARE reporting checklist. Available at http://dx.doi.org/10.21037/ gs-20-386

Conflicts of Interest: All authors have completed the ICMJE uniform disclosure form (available at http://dx.doi. org/10.21037/gs-20-386). The authors have no conflicts of interest to declare.

Ethical Statement: The authors are accountable for all aspects of the work in ensuring that questions related to the accuracy or integrity of any part of the work are appropriately investigated and resolved. All procedures performed in studies involving human participants were in accordance with the ethical standards of the institutional and/or national research committee(s) and with the Helsinki Declaration (as revised in 2013). Written informed consent was obtained from the patient.

Open Access Statement: This is an Open Access article distributed in accordance with the Creative Commons Attribution-NonCommercial-NoDerivs 4.0 International License (CC BY-NC-ND 4.0), which permits the noncommercial replication and distribution of the article with 
the strict proviso that no changes or edits are made and the original work is properly cited (including links to both the formal publication through the relevant DOI and the license). See: https://creativecommons.org/licenses/by-nc-nd/4.0/.

\section{References}

1. Akerström G, Malmaeus J, Bergström R. Surgical anatomy of human parathyroid glands. Surgery 1984;95:14-21.

2. Chen H, Wang TS, Yen TW, et al. Operative failures after parathyroidectomy for hyperparathyroidism: the influence of surgical volume. Ann Surg 2010;252:691-5.

3. Paras C, Keller M, White L, et al. Near-infrared autofluorescence for the detection of parathyroid glands. J Biomed Opt 2011;16:067012.

4. McWade MA, Paras C, White LM, et al. Label-free intraoperative parathyroid localization with near-infrared autofluorescence imaging. J Clin Endocrinol Metab 2014;99:4574-80.

5. McWade MA, Sanders ME, Broome JT, et al. Establishing the clinical utility of autofluorescence spectroscopy for parathyroid detection. Surgery 2016;159:193-202.

6. Ladurner R, Sommerey S, Arabi NA, et al. Intraoperative near-infrared autofluorescence imaging of parathyroid glands. Surg Endosc 2017;31:3140-5.

7. Tsukuura R, Sakai H, Fuse Y, et al. Novel hands-free near-infrared fluorescence navigation and simultaneous

Cite this article as: Aoyama M, Takizawa H, Yamamoto K, Inui T, Miyamoto N, Sakamoto S, Kobayashi T, Uehara H, Tangoku A. Effects of excitation light intensity on parathyroid autofluorescence with a novel near-infrared fluorescence imaging system: two surgical case reports. Gland Surg 2020;9(5):1584-1589. doi: 10.21037/gs-20-386 combined imaging for elevation of vascularized lymph node flap. J Surg Oncol 2018;118:588-9.

8. Seki Y, Kajikawa A, Yamamoto T, et al. Real-time Indocyanine Green Videolymphography Navigation for Lymphaticovenular Anastomosis. Plast Reconstr Surg Glob Open 2019;7:e2253.

9. Riley DS, Barber MS, Kienle GS, et al. CARE guidelines for case reports: explanation and elaboration document. J Clin Epidemiol 2017;89:218-35.

10. Kim SW, Lee HS, Lee KD. Intraoperative real-time localization of parathyroid gland with near infrared fluorescence imaging. Gland Surg 2017;6:516-24.

11. Ladurner $\mathrm{R}$, Lerchenberger $\mathrm{M}, \mathrm{Al}$ Arabi $\mathrm{N}$, et al. Parathyroid Autofluorescence-How Does It Affect Parathyroid and Thyroid Surgery? A 5 Year Experience. Molecules 2019;24:2560.

12. Falco J, Dip F, Quadri P, et al. Cutting Edge in Thyroid Surgery: Autofluorescence of Parathyroid Glands. J Am Coll Surg 2016;223:374-80.

13. Kim SW, Song SH, Lee HS, et al. Intraoperative RealTime Localization of Normal Parathyroid Glands With Autofluorescence Imaging. J Clin Endocrinol Metab 2016;101:4646-52.

14. Kim SW, Lee HS, Ahn YC, et al. Near-Infrared Autofluorescence Image-Guided Parathyroid Gland Mapping in Thyroidectomy. J Am Coll Surg 2018;226:165-72. 